\title{
Distributed Dynamic Traffic Modeling and Implementation Oriented Different Levels of Induced Travelers
}

\author{
Yan Liu ${ }^{1}$ and Yao Yu ${ }^{2}$ \\ ${ }^{1}$ School of Computer Science and Technology, Changchun Normal University, Changchun 130025, China \\ ${ }^{2}$ College of Transport and Communications, Shanghai Maritime University, Shanghai 201306, China \\ Correspondence should be addressed to Yao Yu; yuy_jlu@foxmail.com
}

Received 3 July 2014; Accepted 25 August 2014

Academic Editor: Yongjun Shen

Copyright (C) 2015 Y. Liu and Y. Yu. This is an open access article distributed under the Creative Commons Attribution License, which permits unrestricted use, distribution, and reproduction in any medium, provided the original work is properly cited.

\begin{abstract}
In order to respond to the variable state of traffic network in time, a distributed dynamic traffic assignment strategy is proposed which can improve the intelligent traffic management. The proposed dynamic assignment method is based on utility theory and is oriented to different levels of induced users. A distributed model based on the marginal utility is developed which combines the advantages of both decentralized paradigm and traveler preference, so as to provide efficient and robust dynamic traffic assignment solutions under uncertain network conditions. Then, the solution algorithm including subroute update and subroute calculation is proposed. To testify the effectiveness of the proposed model in optimizing traffic network operation and minimizing traveler's cost on different induced levels, a sequence numerical experiment is conducted. In the experiment, there are two test environments: one is in different network load conditions and the other is in different deployment coverage of local agents. The numerical results show that the proposed model not only can improve the running efficiency of road network but also can significantly decrease the average travel time.
\end{abstract}

\section{Introduction}

Dynamic traffic assignment (DTA) model is the core of traffic guidance system or traffic information service system $[1,2]$. In view of the great time-varying characteristics of traffic network, DTA model can ensure travelers' minimum cost. The aim of applying DTA model is to keep the road network running efficiently. No matter what kind of system applies DTA, the efficiency of calculation is always the key to ensure the effectiveness of this system. According to the analysis of the equilibrium theory, DTA can be divided into two types [3]: one is distributed dynamic traffic assignment (DDTA) and the other is centralized dynamic traffic assignment (CDTA).

Distributed system model has been commonly applied in our daily life, especially in the fields of electricity, energy, manufacture and mass data calculation, and so forth $[4,5]$. Distributed system strategy mainly includes two patterns: the first pattern is an upper decision control pattern, sending dynamic message to each agent which is controlled by central information platform; the second pattern is that each agent can revise the strategy according to the traffic condition in its own area, which can be called local decision.
The latter, namely, the distributed control pattern, can achieve information sharing and communicating among different control agents. However, the control scope of the entire system is composed of multiple subcontrol areas.

\section{Dynamic Traffic Assignment Theory: A Brief Review}

In traffic network, the distributed system is often used in the fields of path searching, traffic signal control, and so on, such as solving TSP problem $[6,7]$, vehicle navigation, and intersection signal control. Guo and Huang [8] proposed an optimal control method with feedback adjustment. This method was devoted to forecasting the traffic state at the next moment by loading queue length and the remaining road capacity on intersection node and was applied in one-way OD matrix. Papageorgiou [9] presented a feedback optimal control model for time-varying traffic flow. This model is considered to be a dynamic, macroscopical, and deterministic mathematical model, which can achieve the dynamic user equilibrium. The stability of this model is poor under nonlinear traffic 
problems (such as traffic congestion), although its inner parameters were calibrated by repeated experiments. Kuwahara and Akamatsu [10] proposed a time-dependent dynamic assignment model with multi-OD matrix. In their assumption, vehicles make decisions according to instantaneous travel time and follow the principles of flow conservation and FIFO. But the numerical experiments were not discussed.

Hawas [11] presented a distributed route guidance strategy based on real-time path searching, in which it is assumed that each node (intersections) in network acts as an agent and the responsible area of each agent depends on the depth of knowledge. That is to say, the greater the processing capacity of node information is, the lager the responsible area will be. Distributed processing is different from the centralized mode. The former is allowed to use the current information in itself's area and choose the alternative paths to make evaluation in order to pick the better suitable subpath. As shown in Figure 1, the area is composed of nodes (intersections) and road segments. The control territory by agents is less than or equal to their own depth knowledge.

As shown in Figure 1, the responsible area of decision agent is a circle in which $r=2$. Hawas put forward four path assignment principles, which are, respectively, (1) to evaluate path by travel time estimation, where vehicles will be assigned to the subpath with minimum travel time; (2) to equally evaluate path by travel time estimation, where vehicles will be divided into several groups and assigned to different subpaths, respectively; (3) to evaluate path by the generalized evaluation function which contains multiple parameters (such as marginal travel time); (4) to allow the information interaction and sharing among the agents and to evaluate alternative paths by the marginal travel time.

\section{Models}

Traditional methods cannot solve the problem of dynamic traffic assignment under the large-scale urban road network, which promote the emergence and development of distributed dynamic traffic assignment. Different from the centralized control which requires highly, in respect of integrity of input data, high complexity of valgorithms and time effectiveness of computation, the distributed architecture provides a real-time self-adaptive assignment strategy which is based on limited local information. Such strategies based on local control can effectively respond to real-time changes in network traffic and are small in calculation amount and fast in updating speed. Particularly when network traffic incident occurs or O-D predictive value is not accurate, the validity of distributed guidance strategy is superior to CDTA.

Travelers do not fully accept and implement the information which is obtained from the center or the client. These choice differences are reflected by the limited rational behavior in their travelling. According to the existing research with respect to driver classification as proposed by Fan and Machemehl and so forth [12], we can divide the user group who accept guidance information into the following four types:

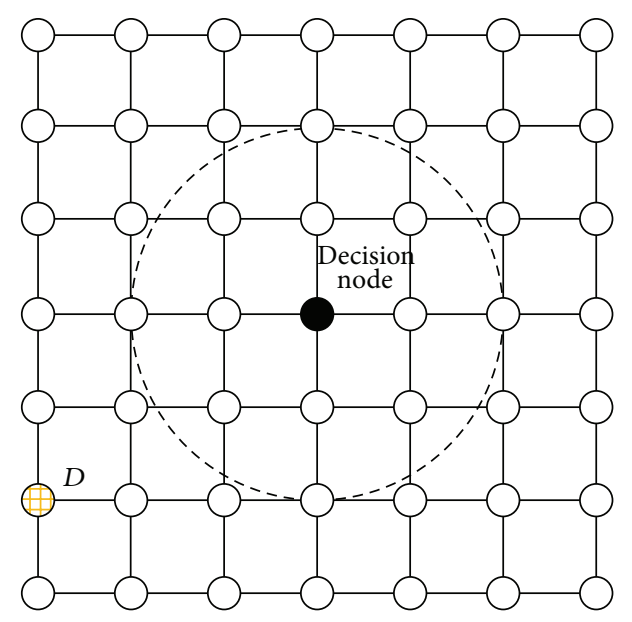

FIGURE 1: Control territory by agent when knowledge depth is 2 .

(1) level 1, the driver whose car is not equipped with the vehicle navigation apparatus travels in accordance with preset route by relying on historical travel information or outside information that was once received;

(2) level 2, the driver whose car is equipped with car navigation equipment travels by following the prescribed route sent by the system/center;

(3) level 3, the driver whose car is equipped with car navigation equipment travels by following the routine under user optimal;

(4) level 4, drivers with bounded rationality change the travel route based on the information obtained in real time.

This study assumes that, under the circumstance that only part of the OD travel demand is known, DDTA certainly cannot achieve the goal of minimum travel time or lowest expenditure in a general situation. Moreover, it assumes that the road network has several agents and any of the agents within its regional "jurisdiction" is responsible for the development of inducing strategy for the entering vehicles. The size of the area is restricted by computational performance, accuracy requirements, and other conditions. By inputting limited information, the optional path $(\mathrm{O}-\mathrm{D})$ is evaluated and the vehicle (instantaneous distribution) is distributed to the node in the downstream segment. The agents make decisions by analyzing the advantages and disadvantages and use local state (local area, 11 ) and nonlocal (nonlocal, nl) state to present.

(1) The former describes the current state of the path within the area.

(2) The latter expresses that the expected status exceeds the level of knowledge $\pi$.

Through the road network segmentation, driving route of the vehicle can be seen as a series of subpath selections. Each agent point is a decision point, which can use the known 
information and heuristic methods to evaluate the subpath sets that we can choose and to feed back the results to the entering vehicles.

To define subpath $k \in K(l, n, j, t, \pi), k$ means subpaths from the decision point $n$ to the end point $j$, when the knowledge depth of agent is $\pi$ and the vehicle is subjected to the conditions of level $l$ at the time of $t$. The value of $\pi$ is determined according to the scope of extractable information from the downstream segment (or nodes). As shown in Figure 2, the knowledge depth $\pi$ of agent point at $n$ is 2 .

Explanations on model symbols are as follows:

$l r_{n, j}^{t}$ : the number of vehicles which is distributed to subpaths $k \in K(l, n, j, t, \pi)$ by agent from agent point $n$ to the end point $j$ at the time of $t$;

$q_{n, j, k}^{t}$ : the total number of vehicles which is in subpaths $k \in K(l, n, j, t, \pi)$ from agent point $n$ to the end point $j$ at the time of $t$;

$J$ : collection at destination;

$N$ : collection at agent point;

$\Gamma$ : time, $\Gamma=\left[t_{0}, t_{e}\right]$;

$K(l, n, j, t)$ : the collection of subpaths, when the user's level is $l$ and the user has accepted induction from point $n$ to the end point $j$ at the time of $t$;

$t_{0}$ : the start time;

$t_{e}$ : the end time;

$T_{n, j, k}^{t}$ : the travel time of path $k, k \in K(l, n, j, t, \pi)$ from point $n$ to the end point $j$;

$T_{n, j, k}^{t, l l}$ : the real travel time of path $k, k \in K(l, n, j, t, \pi)$ from point $n$ to the end point $j$ in local state (ll);

$T_{n, j, k}^{t, \mathrm{nl}}$ : the real travel time of path $k, k \in K(l, n, j, t, \pi)$ from point $n$ to the end point $j$ in nonlocal state (nl).

When $t \in \Gamma$ and agent $n \in N$, it is mathematically expressed as follows:

$$
\min _{l r_{n, j, k}^{t}} Z_{n, j}^{t}=\sum_{j} \sum_{k \in K(l, n, j, t)}\left[\left(q_{n, j, k}^{t}+l r_{n, j, k}^{t}\right) T_{n, j, k}^{t}\right]
$$

s.t.

$$
\begin{gathered}
\sum_{k \in K(l, n, j, k)} l r_{n, j, k}^{t}=l r_{n, j, k}^{t} \quad \forall j, \\
T_{n, j, k}^{t}=T_{n, j, k}^{t, l l}+T_{n, j, k}^{t, n l} \quad \forall j, k \in K(l, n, j, t), \\
l r_{n, j, k}^{t} \geq 0 .
\end{gathered}
$$

The objective function equation (1) represents that, within the agent coverage, the function of the vehicle travel time is minimum; the constraints function (2) is flow conservation constraints, which represents that the total sum of flows allocated to each path is equal to the total sum of vehicles that have left the agent; the constraints equation (3) represents the travel time from point $n$ to the end point $j$, which includes the travel time in local state of subpath and the travel time

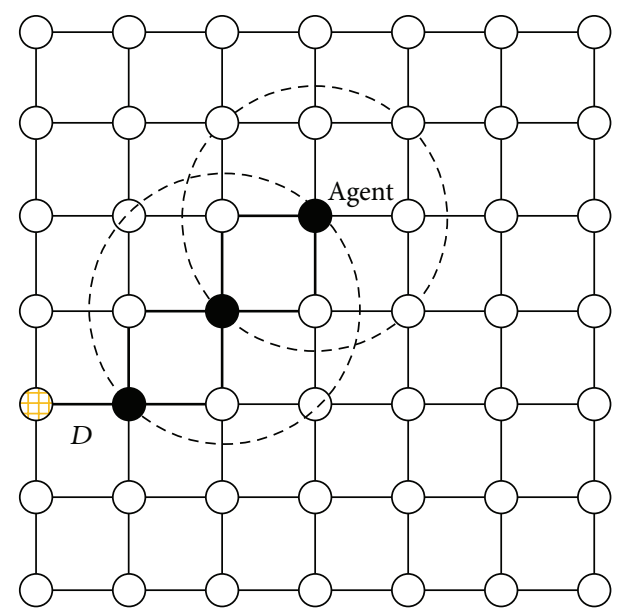

FIGURE 2: DDTA agent control territory.

in nonlocal state of subpath; the constraints equation (4) represents that the number of vehicles is nonnegative.

According to the Lagrange equation, it can be known that (1) and (2) can be rewritten as

$$
\begin{aligned}
L= & \sum_{k \in K(l, n, j, k)}\left\{\left(q_{n, j, k}^{t}+l r_{n, j, k}^{t}\right) T_{n, j, k}^{t}\right\} \\
& -\delta_{n, j}^{t}\left(\sum_{k \in K(l, n, j, k)} l r_{n, j, k}^{t}-l r_{n, j}^{t}\right),
\end{aligned}
$$

where $\delta_{n, j}^{t}$ is the Lagrangian arithmetic operator and meets the constraint condition for the conservation of demand.

$\mathrm{K}-\mathrm{K}-\mathrm{T}$ (Karush-Kuhn-Tucker) is optimized as follows:

$$
\begin{array}{r}
\frac{\partial L}{\partial l r_{n, j, k}^{t}}=\frac{\partial l r_{n, j, k}^{t} T_{n, j, k}^{t}}{\partial l r_{n, j, k}^{t}}-\delta_{n, j}^{t} \\
=T_{n, j, k}^{t}+l r_{n, j, k}^{t} \frac{\partial T_{n, j, k}^{t}}{\partial l r_{n, j, k}^{t}}-\delta_{n, j}^{t} \\
\forall j, t, k \in K(l, n, j, \pi) ;
\end{array}
$$

then

$$
\begin{array}{r}
l r_{n, j, k}^{t} \frac{\partial L}{\partial l r_{n, j, k}^{t}}=l r_{n, j, k}^{t}\left(T_{n, j, k}^{t}+l r_{n, j, k}^{t} \frac{\partial T_{n, j, k}^{t}}{\partial l r_{n, j, k}^{t}}-\delta_{n, j}^{t}\right)=0 \\
\forall j, t, k \in K(l, n, j, \pi) .
\end{array}
$$

When $l r_{n, j, k}^{t} \geq 0$, the path margin travel time is the minimum at $\delta_{n, j}^{t}$. $T_{n, j, k}^{t}$ can be substituted with $T_{n, j, k}^{t, l}$ in (6), which means real-time travel in local region; similarly, in the nonlocal region, real travel time can be expressed as $T_{n, j, k}^{t, n l}$ :

$$
\frac{\partial L}{\partial l r_{n, j, k}^{t}}=\left(T_{n, j, k}^{t, l}+T_{n, j, k}^{t, \mathrm{nl}}\right)+\operatorname{lr} r_{n, j, k}^{t} \frac{\partial\left(T_{n, j, k}^{t, l}+T_{n, j, k}^{t, \mathrm{nl}}\right)}{\partial l r_{n, j, k}^{t}}-\delta_{n, j}^{t} \geq 0 .
$$


And the total margin travel time is defined as follows:

$$
H_{n, j, k}^{t}=\left(T_{n, j, k}^{t, l}+T_{n, j, k}^{t, n l}\right)+l r_{n, j, k}^{t} \frac{\partial\left(T_{n, j, k}^{t, l}+T_{n, j, k}^{t, n l}\right)}{\partial l r_{n, j, k}^{t}},
$$

where $H_{n, j, k}^{t}$ represents the real marginal travel time of path $k$ from point $n$ to the end point $j$ at $t$ time.

The marginal travel time is $\partial T_{n, j, k}^{t, l} / \partial l r_{n, j, k}^{t}$ in the range of the agent, but the marginal travel time is $\partial T_{n, j, k}^{t, n l} / \partial l r_{n, j, k}^{t}$ in nonlocal state. Equation (10) represents the marginal travel time of path $k$ from point $n$ to the end point $j$ in nonlocal state:

$$
\begin{aligned}
H_{n, j, k}^{t} & =M_{n, j, k}^{t, l}+M_{n, j, k}^{t, \mathrm{nl}} \\
& =\left(T_{n, j, k}^{t, l}+l r_{n, j, k}^{t} \frac{\partial T_{n, j, k}^{t, l}}{\partial l r_{n, j, k}^{t}}\right)+\left(\frac{\widetilde{S}_{n, j, k}^{t, n l}}{S_{n, j, k}^{t, l}}\right) T_{n, j, k}^{t, l} \\
& =\left(1+\frac{\widetilde{S}_{n, j, k}^{t, n l}}{S_{n, j, k}^{t, l}}\right) T_{n, j, k}^{t, l}+l r_{n, j, k}^{t} \frac{\partial T_{n, j, k}^{t, l}}{\partial l r_{n, j, k}^{t}},
\end{aligned}
$$

where $H_{n, j, k}^{t}$ is the total marginal travel time of path $k$ from point $n$ to the end point $j ; M_{n, j, k}^{t, l}$ is the marginal travel time of path $k$ from point $n$ to the end point $j$ in local state; $M_{n, j, k}^{t, n l}$ is the marginal travel time of path $k$ from point $n$ to the end point $j$ in nonlocal state; $S_{n, j, k}^{t, l}$ is length ratio of path $k$ in local state; $\widetilde{S}_{n, j, k}^{t, n l}$ is length ratio of path $k$ in nonlocal state.

Comparing (10) with (9), the difference of second type is the error between the exact marginal travel time and the estimated marginal travel time, which is

$$
\begin{aligned}
\Delta & =\left(T_{n, j, k}^{t, l}-\frac{\widetilde{S}_{n, j, k}^{t, \mathrm{nl}}}{S_{n, j, k}^{t, l}} T_{n, j, k}^{t, l}\right)+l r_{n, j, k}^{t} \frac{\partial T_{n, j, k}^{t, l}}{\partial l r_{n, j, k}^{t}} \\
& =\left(T_{n, j, k}^{t, l}+r_{n, j, k}^{t} \frac{\partial T_{n, j, k}^{t, l}}{\partial l r_{n, j, k}^{t}}\right)-\frac{\widetilde{S}_{n, j, k}^{t, n l}}{S_{n, j, k}^{t, l}} T_{n, j, k}^{t, l} .
\end{aligned}
$$

Equation (11) shows that when accurate marginal travel time approaches the estimated time and the length ratio in local state and the length ratio in nonlocal state are approximate, the error is zero. However, due to traffic conditions and time-varying characteristics of travel demand, the error would hardly disappear.

In order to reduce the error between the actual value and the estimated value, this study proposes the introduction of the shortest path information as calculated in the vehicle system and applies the same in the local road network which is covered by the agent to estimate the marginal travel time in nonlocal area. That is to say, (10) can be rewritten as

$$
\begin{aligned}
H_{n, j, k}^{t} & =M_{n, j, k}^{t, l}+M_{n, j, k}^{t, \mathrm{nl}} \\
& =\left(T_{n, j, k}^{t, l}+l r_{n, j, k}^{t} \frac{\partial T_{n, j, k}^{t, l}}{\partial l r_{n, j, k}^{t}}\right)+T_{n, j, k}^{t, \mathrm{nl}},
\end{aligned}
$$

where $l r_{n, j, k}^{t}\left(\partial T_{n, j, k}^{t, l} / \partial l r_{n, j, k}^{t}\right)$ represent the error between the actual value and the estimated value. As it can be seen from the error term, the marginal travel time valuated in the model is low, because we assumed that travelers' behavior was known. With increasing distance (the farther away from the decision-making agent), its additional marginal influences on path decision-making became smaller. So there is $\partial T_{n, j, k}^{t, l} / \partial l r_{n, j, k}^{t} \rightarrow 0$.

\section{Model Solution}

The solving process of the DDTA (marginal-based DDTA, M-DDTA) model based on marginal travel time in the above section is divided into the following two parts: (1) the generation of agent subpath sets and (2) the update of subpath sets.

This paper puts forward subpath generation algorithm (SGA) for the first part. Agent calculates the local alternative paths in the network with the purpose of selecting the feasible subpath sets. In the selection process, this paper takes traveler (user) factor into consideration, such as $\pi$ which represents the depth of knowledge. Alternative subpath sets do not change in the execution of agent.

The focus of the second algorithm part is renewing the subpath set which is called subpath updating algorithm (SUA) in this paper. The object of update is the subpath set which is established by SGA in the first step. SUA estimates the travel time of the nonlocal area by heuristic search [12] or directly receives the marginal travel time information transmitted by the center, in order to meet the requirements for time efficiency of the route guidance calculation.

4.1. SGA Algorithm. Firstly, SGA sets agent as "root node" and generates all the subpaths within its scope. Then, a subpath list will be established, and all the subpaths in the list will be reviewed and those invalid paths will be removed. Finally, a feasible subpath set will be formed and provided to SUA algorithm in the second step for the update of subpath set.

The specific steps of SGA are as follows.

Step 1. Prepare the expression of road network structure (the forward star structure).

Step 2. Create scan path table $S_{n}^{\pi}$; the scan starting point is the agent point $n$ and the scan area is determined by the depth of knowledge $\pi$.

Step 2.1. Initialization. Define a starting position of the pointer array $c=1$ and the counter $d=0$. Make $\left|S_{\pi}^{n}\right|=1$, $S_{\pi}^{n}(c)=n$, and the depth $D(c)=0$, as well as the precursor $p\left[S_{\pi}^{n}(c)\right]=0$.

Step 2.2. Make $d=d+1$. For arbitrary point $o=S_{\pi}^{n}(c)$, add nodes $m \in a(o)$ in which $a(o)$ represents adjacent nodes set of node $o$ in the table. But at the bottom of the form, make $m \neq p(o)$. Make precursor $p(m)=0, D(m)=d$, and $c=c+1$.

Step 2.3. If $d=\pi+1$, stop traversal; otherwise repeat Step 2.1 and Step 2.2. 
Step 3. Construct alternative paths according to the table $S_{n}^{\pi}$.

Step 3.1. Initialize the path set, making the path count $k=$ $1, c=\left|S_{\pi}^{n}\right|$.

Step 3.2. Initialize the path $k$, making continuous node count $j=1$ and node label $n_{j}=S_{\pi}^{n}(c)$.

Step 3.3. Set $j=j+1$.

Step 3.4. Set $n_{j}=p\left[S_{\pi}^{n}(c)\right]$.

Step 3.5. If $p\left(S_{c}\right)=0$, then stop; otherwise repeat Step 3.3 to Step 3.5.

Step 3.6. Set $k=k+1$ and $c=c-1$.

Step 3.7. If $c=0$, then stop; otherwise repeat Step 3.2 to Step 3.6.

Step 4. Remove the invalid path in the table $S_{n}^{\pi}$ and ensure the subpath $k$ does not include loop.

4.2. SUA Algorithm. The subpath set $S_{n}^{\pi}$ generated by SGA does not change in the agent decision process, which is updated only when the road network topology changes or the agent changes itself. In order to guarantee the rationality of the subpath distribution, each agent in the road network needs to calculate the total marginal travel time (as shown in (10)). The total marginal travel time of a path consists of two parts, which are, respectively, local marginal travel time estimation and nonlocal marginal travel time estimation, as represented in the equation $H_{n, j, k}^{t}=M_{n, j, k}^{t, l}+M_{n, j, k}^{t, n l}$. Local travel time can be collected through road-side traffic flow detector, while marginal travel time can only be obtained by computing the corresponding estimation values. This paper uses Greenberg model [13] to estimate local marginal travel time:

$$
u_{a}^{t}=u_{a}^{o} \ln \left(\frac{k_{a}^{\mathrm{jam}}}{k_{a}^{t}}\right)
$$

where $u_{a}^{t}$ denotes the speed on the road $a$ at the time of $t ; u_{a}^{o}$ denotes the free-flow speed on the road $a ; k_{a}^{\mathrm{jam}}$ denotes the jam density on the road $a ; k_{a}^{t}$ denotes the density on the road $a$ at the time of $t$.

The real-time travel time on the road $a$ can be obtained by using the above equation. In fact, this travel time is a function of traffic flow. The traffic flow contains the existing vehicles on the road $a$ at the time of $t$ and the number of vehicles running into road $a$. The equation is as follows:

$$
t_{a}^{t}\left(x_{a}^{t}+r_{n, j, a}^{t}\right)=\frac{d_{a}}{u_{a}^{t}}=\frac{d_{a}}{u_{a}^{t}} \ln \left(\frac{k_{a}^{\mathrm{jam}}}{k_{a}^{t}}\right)^{-1},
$$

where $d_{a}$ is length of $\operatorname{road} a$.
To calculate the partial derivative of $r_{n, j, k}^{t, l}$ for $t_{a}^{t}\left(x_{a}^{t}+r_{n, j, a}^{t}\right)$, the above equation becomes as follows:

$$
\begin{aligned}
\frac{\partial t_{a}^{t}\left(x_{a}^{t}+r_{n, j, a}^{t, l}\right)}{\partial r_{n, j, a}^{t, l}} & =\frac{\partial t_{a}^{t}\left(x_{a}^{t}+r_{n, j, a}^{t, l}\right)}{\partial k_{a}^{t}} \frac{\partial k_{a}^{t}}{\partial r_{n, j, a}^{t, l}} \\
& =\frac{d_{a}}{u_{a}^{o}\left(x_{a}^{t}+r_{n, j, a}^{t, l}\right)} \ln \left(\frac{k_{a}^{\mathrm{jam}}}{k_{a}^{t}}\right)^{-2} .
\end{aligned}
$$

Therefore, the marginal travel time $M_{n, j, k}^{t, l}$ of subpath $k$ in local area is given below:

$$
\begin{aligned}
& M_{n, j, k}^{t, l}=\sum_{a \in k}\left[\left(x_{a}^{t}+r_{n, j, a}^{t, l}\right) \frac{\partial t_{a}^{t}\left(x_{a}^{t}+r_{n, j, a}^{t, l}\right)}{\partial r_{n, j, a}^{t, l}}+t_{a}^{t}\left(x_{a}^{t}+r_{n, j, a}^{t, l}\right)\right] \\
& =\sum_{a \in k} \frac{d_{a}}{u_{a}^{o}}\left\{\left[\ln \left(\frac{k_{a}^{\mathrm{jam}}}{k_{a}^{t}}\right)^{-2}\right]+t_{a}^{t}\left(x_{a}^{t}+r_{n, j, a}^{t, l}\right)\right\} \\
& =\sum_{a \in k} \frac{d_{a}}{u_{a}^{o}}\left\{\left[\ln \left(\frac{k_{a}^{\mathrm{jam}}}{k_{a}^{t}}\right)^{-2}\right]+\left[\ln \left(\frac{k_{a}^{\mathrm{jam}}}{k_{a}^{t}}\right)^{-1}\right]\right\} \text {. }
\end{aligned}
$$

It can be seen that it is enough to obtain the traffic density $k_{a}^{t}$ only. Other variables such as free-flow speed, the length of road section, and jam density are constant.

The nonlocal marginal travel time can be calculated according to (10). Euclidean distance is used to estimate $S_{n, j, k}^{t, l}$ and $\widetilde{S}_{n, j, k}^{t, n l}$. When the road network structure is regular grid, Manhattan distance [14] is more appropriate. The equation of Euclidean distance is as follows:

$$
\begin{aligned}
& S_{n, j, k}^{t, l}=\sqrt{\left(x x_{n}-x x_{z}\right)^{2}+\left(y y_{n}-y y_{z}\right)^{2}}, \\
& \widetilde{S}_{n, j, k}^{t, l}=\sqrt{\left(x x_{z}-x x_{j}\right)^{2}+\left(y y_{z}-y y_{n}\right)^{2}},
\end{aligned}
$$

where $\left(x x_{n}, y y_{n}\right)$ denotes $x-y$ coordinates of the $n$ point; $\left(x x_{z}, y y_{z}\right)$ denotes $x-y$ coordinates of the end point $z$ in subpath; $\left(x x_{j}, y y_{j}\right)$ denotes $x-y$ coordinates of the destination point $j$.

The equation of Manhattan distance is as follows:

$$
\begin{aligned}
& S_{n, j, k}^{t, l}=\left|x x_{n}-x x_{z}\right|+\left|y y_{n}-y y_{z}\right|, \\
& \widetilde{S}_{n, j, k}^{t, l}=\left|x x_{z}-x x_{j}\right|+\left|y y_{z}-y y_{n}\right|,
\end{aligned}
$$

where $\left(x x_{n}, y y_{n}\right)$ denotes $x-y$ coordinates of the $n$ point; $\left(x x_{z}, y y_{z}\right)$ denotes $x-y$ coordinates of the end point $z$ in subpath; $\left(x x_{j}, y y_{j}\right)$ denotes $x-y$ coordinates of the destination point $j$.

Considering the distance between nonlocal area and local decision point, $T_{n, j, k}^{t, \mathrm{nl}}+l r_{n, j, k}^{t}\left(\partial T_{n, j, k}^{t, \mathrm{nl}} / \partial l r_{n, j, k}^{t}\right)$ can be simplified as $T_{n, j, k}^{t, n l}$, when it is assumed that the impact of marginal travel time can be ignored when the subpath $k$ adds one car. 


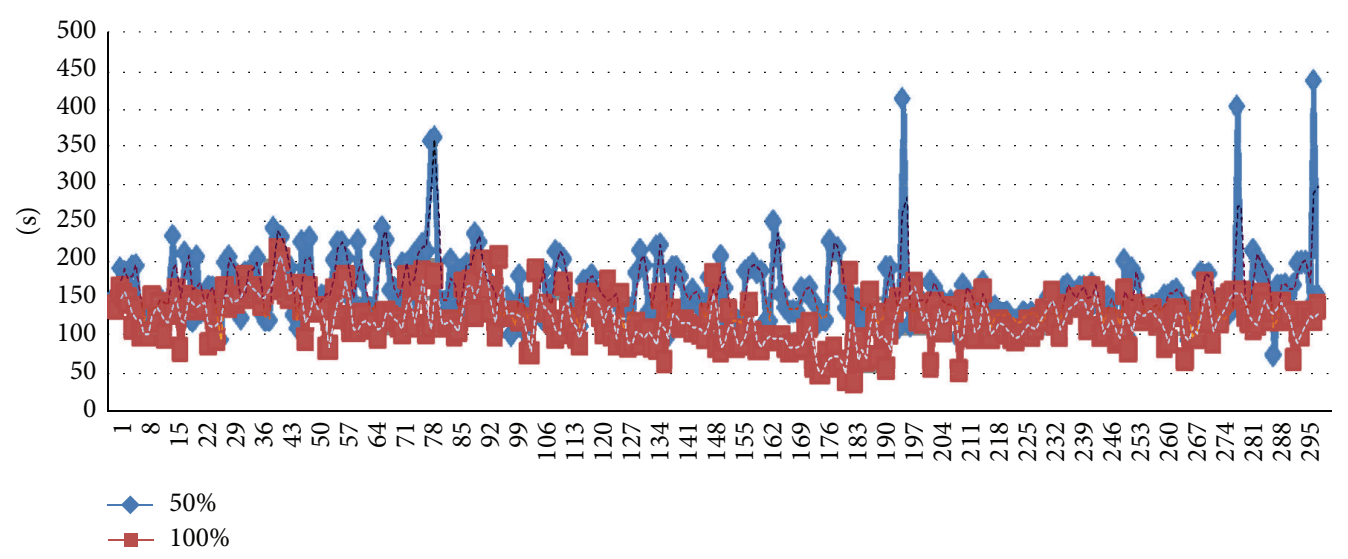

FIGURE 3: Comparison of average travel time under 9800 veh network loading.

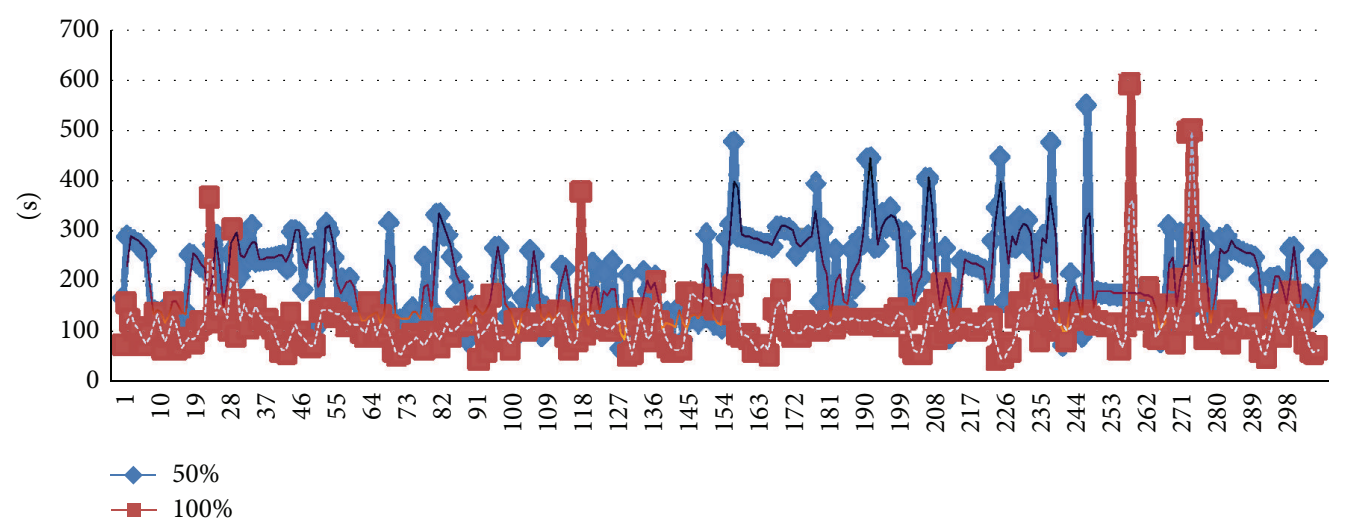

Figure 4: Comparison of average travel time under 12200 veh network loading.

According to the Logit model which is proposed by Hawas, we can obtain $P_{k}$ as follows:

$$
P_{k}=\frac{e^{-\alpha H_{k}}}{\sum_{i} e^{-\alpha H_{i}}},
$$

where $P_{k}$ denotes the probability of vehicles being assigned to the subpath $k ; H_{k}$ denotes the total marginal travel time of subpath $k ; \alpha$ is adjustment coefficient of the total marginal travel time $H_{k}$.

The $\alpha$ can adjust the distribution proportion of traffic flow indifferent paths. Therefore, the value of $\alpha$ directly affects the actual traffic flow of the path. Assuming there are two paths denoted as $k_{1}$ and $k_{2}$, the distribution proportion is $P_{k_{1}} / P_{k_{2}}=$ $e^{-\alpha\left(H_{k 1}-H_{k 2}\right)}$ according to (19). As $\alpha$ increases, the difference of the total marginal travel time between two paths will be greater.

Specific SUA steps are as follows.

Step 1. Calculate the total marginal travel time $H_{n, j, k}^{t}$ of all paths $k$ when $k \in(l, n, j, t, \pi)$. The local marginal travel time $M_{n, j, k}^{t}$ is calculated by using (16). The nonlocal marginal travel time is estimated by using Euclidean distance or Manhattan distance according to different road network structures.
Step 2. Calculate the distribution proportion of different subpath $k, k \in(l, n, j, t, \pi)$ according to (4)-(19).

Step 3. Distribute the vehicle which leaves at the time of $t$ from node $n$ to the destination $j$.

Step 4. Repeat Step 1 to Step 3.

\section{Numerical Analysis}

The agent deployment principles under distributed guidance environment are determined by the following two points: (1) topology of road network and (2) distribution of crowding/congestion in road network. The percentage of deployment coverage of agents actually determines the effect of guidance. The coverage of road network (nodes) has a direct impact on the development and update of DTA strategy.

This study adapted full coverage and partial coverage modes, respectively, and a parameter (average travel time) was simulated under various traffic states. The numerical results are shown in Figures 3, 4, and 5.

In Figures 3-5, the horizontal axis represents the vehicle number and the vertical axis represents the average travel time. Traffic flow loading in Figures 3 to 5 is 9800 veh, $12200 \mathrm{veh}$, and $17000 \mathrm{veh}$, respectively. The blue curve and 


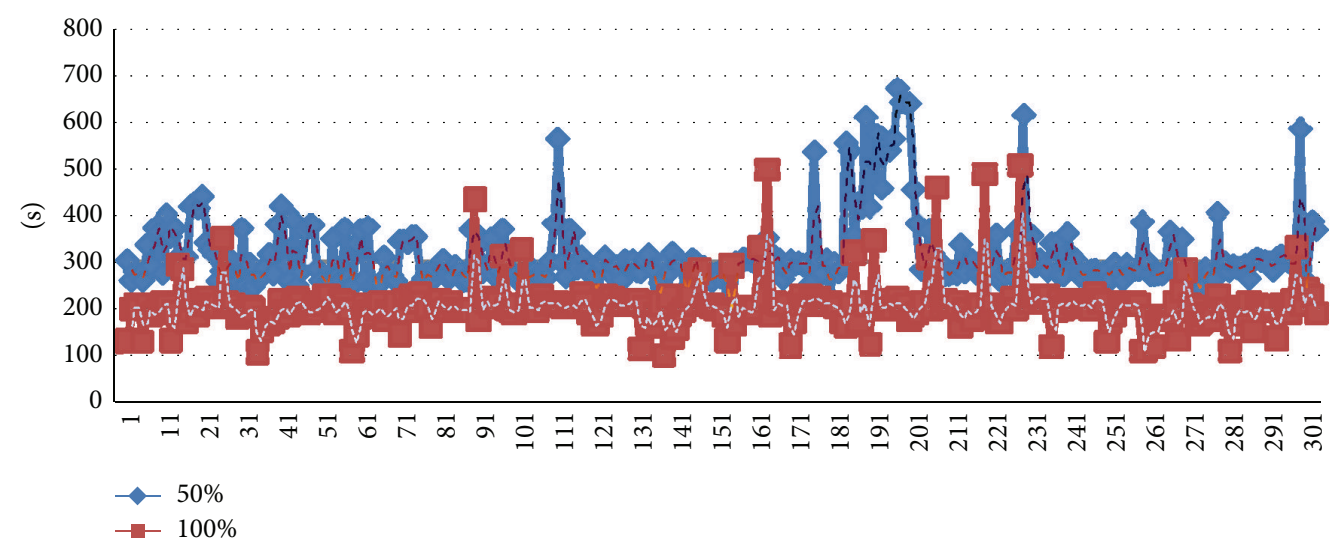

FIGURE 5: Comparison of average travel time under 17000 veh network loading.

red curve represent that the deployment coverage is $50 \%$ and $100 \%$, respectively. As mentioned earlier, the agents' coverage of the road network has a significant impact on network equilibrium assignment. So the links travel time will be increasing with the deployment coverage decreasing. On the contrary, the efficiency of network will become better with the percentage increments. These results also show the importance of deploying the agents rationally.

According to Figure 3, it can be known that when the network load is low, the traffic flow is relatively small and the average vehicle speed is relatively high, and thus the average travel time is less. When agent deployment coverage increases from $50 \%$ to $100 \%$, the average travel time shows a significant decrease of about $15 \%$. When the network load environment is $122000 \mathrm{veh}$, the links become a slight congestion and the average travel time increases. In contrast with $50 \%$ deployment coverage, with $100 \%$ deployment coverage, the average travel time decreases by about $21 \%$. In this scenario, the average travel is more than that in the first experiment. Figure 5 shows a sharp increase comparing with the other scenarios. Apparently, the curve descriptive $100 \%$ coverage is better than the $50 \%$ coverage curve. The travel time decreased approximately by $20 \%$. No matter in what situations the experiments are conducted, the curves all present similar decreasing tendency. Namely, traffic information sharing and communicating among the agent control territories are the basis of the efficiency of DDTA. Moreover, the more the percentage of deployment coverage, the more the efficiency and timeliness with the DDTA running.

\section{Conclusion}

This study proposed a new traffic guidance strategy. Different from the traditional traffic assignment process, the proposed method classifies the levels of road users according to the analysis of their actual inducing requirement on the basis of the expected utility theory research at the beginning and then it partitions the road network into multiple subareas which are controlled by their local agents. This novel dynamic assignment strategy not only considers the actual traveler's preference, but also can achieve a rapid response to the emergency incident in local area. Aiming to verify the model of dynamic traffic assignment based on marginal travel time and its solution algorithm, a set of scenarios are also designed to investigate the performance of road network. Consequently, a series of meaningful results were obtained, where it appears that the deployment coverage of local agent becomes a restricting factor for the system guidance performance. With road network load increments, the application of the proposed method can effectively reduce the vehicles average travel time by more than $20 \%$.

\section{Conflict of Interests}

The authors declare that there is no conflict of interests regarding the publication of this paper.

\section{Acknowledgments}

The authors express his sincere appreciation to the "12th FiveYear Plan" Science and Technology Research of Educational Office of JiLin Province for the financial supports of this subject under Grant no. 261 [2013] and Natural Science Foundation of Changchun Normal University under Grant no. 004 [2014].

\section{References}

[1] Y. Zhou, X. Yang, and M. Chou, "Dynamic route guidance based on model predictive control," Computer Modeling in Engineering and Sciences, vol. 92, no. 5, pp. 477-491, 2013.

[2] Y. Sheffi, Urban Transportation Networks: Equilibrium Analysis with Mathematical Programming Methods, Prentice Hall, New York, NY, USA, 1985.

[3] Q. Guan, Research on the theory and key technologies of cooperation between region traffic signal control and traffic guidance [Ph.D. dissertation], JiLin University, Changchun, China, 2009.

[4] B. J. Binder, J. Benjamina, K. A. Landman et al., "Quantifying evenly distributed states in exclusion and nonexclusion processes," Physical Review E-Statistical, Nonlinear, and Soft Matter Physics, vol. 83, no. 4, Article ID 041914, pp. 1-9, 2011. 
[5] L. Gaol and Z. Zhang, "Management of pavement maintenance, rehabilitation, and reconstruction through network partition," Transportation Research Record, vol. 2366, pp. 59-63, 2013.

[6] Z.-S. Yang, Y. Yu, D.-X. Yu, H.-X. Zhou, and X.-L. Mo, "APFbased car following behavior considering lateral distance," Advances in Mechanical Engineering, vol. 2013, Article ID 207104, 8 pages, 2013.

[7] D.-X. Yu, Z.-S. Yang, Y. Yu, and X.-R. Jiang, "Research on large-scale road network partition and route search method combined with traveler preferences," Mathematical Problems in Engineering, vol. 2013, Article ID 950876, 8 pages, 2013.

[8] R. Y. Guo and H. J. Huang, "Joint optimization model of roaduse pricing and capacity using the optimal control theory ," Journal of Transportation Systems Engineering and Information Technology, vol. 7, no. 6, pp. 61-66, 2007.

[9] M. Papageorgiou, "Dynamic modeling, assignment, and route guidance in traffic networks," Transportation Research B, vol. 24, no. 6, pp. 471-495, 1990.

[10] M. Kuwahara and T. Akamatsu, "Dynamic user optimal assignment with physical queues for a many-to-many OD pattern," Transportation Research B, vol. 35, no. 5, pp. 461-479, 2001.

[11] Y. Hawas, A decentralized architecture and local search procedures for real-time route guidance in congested vehicular traffic network [Dissertation], The University of Texas at Austin, 1996.

[12] W. Fan and R. B. Machemehl, "Tabu search strategies for the public transportation network optimizations with variable transit demand," Computer-Aided Civil and Infrastructure Engineering, vol. 23, no. 7, pp. 502-520, 2008.

[13] C. S. Putcha, R. R. Tadi, M. Charoensuphong, and J. H. Kreiner, "Development of improved and efficient traffic flow model," International Journal of Modelling and Simulation, vol. 27, no. 1, pp. 89-93, 2007.

[14] T. Sherwood, E. Perelman, G. Hamerly, and B. Calde, "Automatically characterizing large scale program behavior," $A C M$ SIGARCH Computer Architecture News, vol. 30, no. 5, pp. 4557, 2002. 


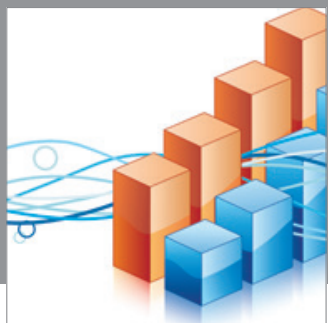

Advances in

Operations Research

mansans

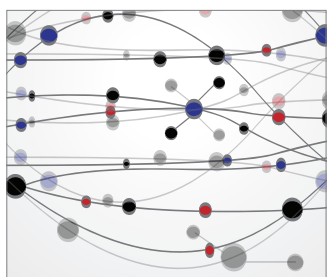

The Scientific World Journal
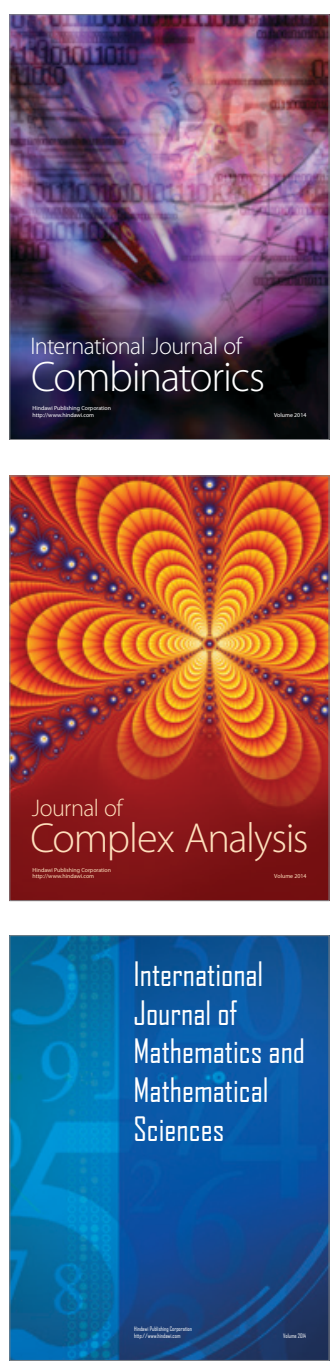
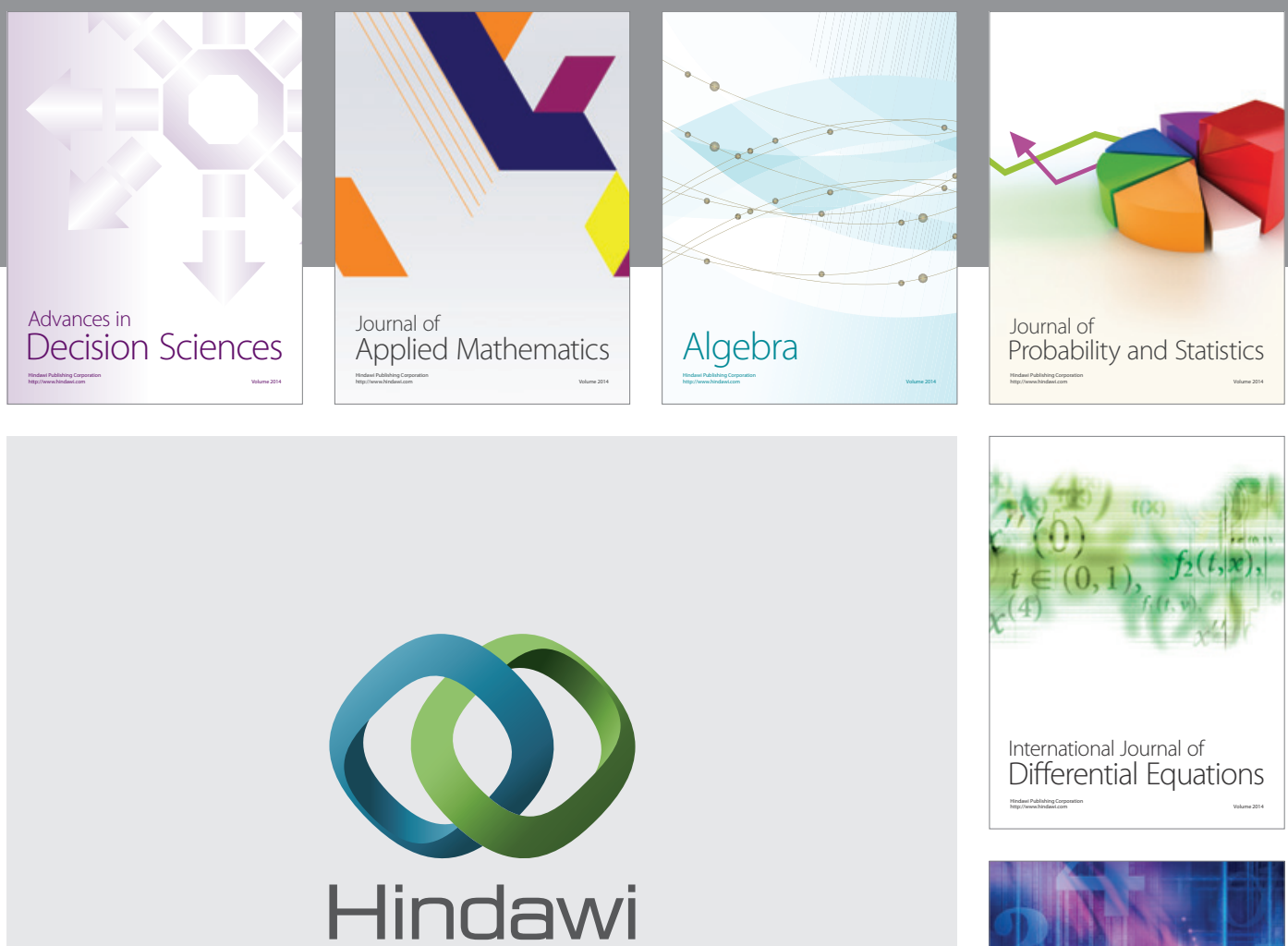

Submit your manuscripts at http://www.hindawi.com
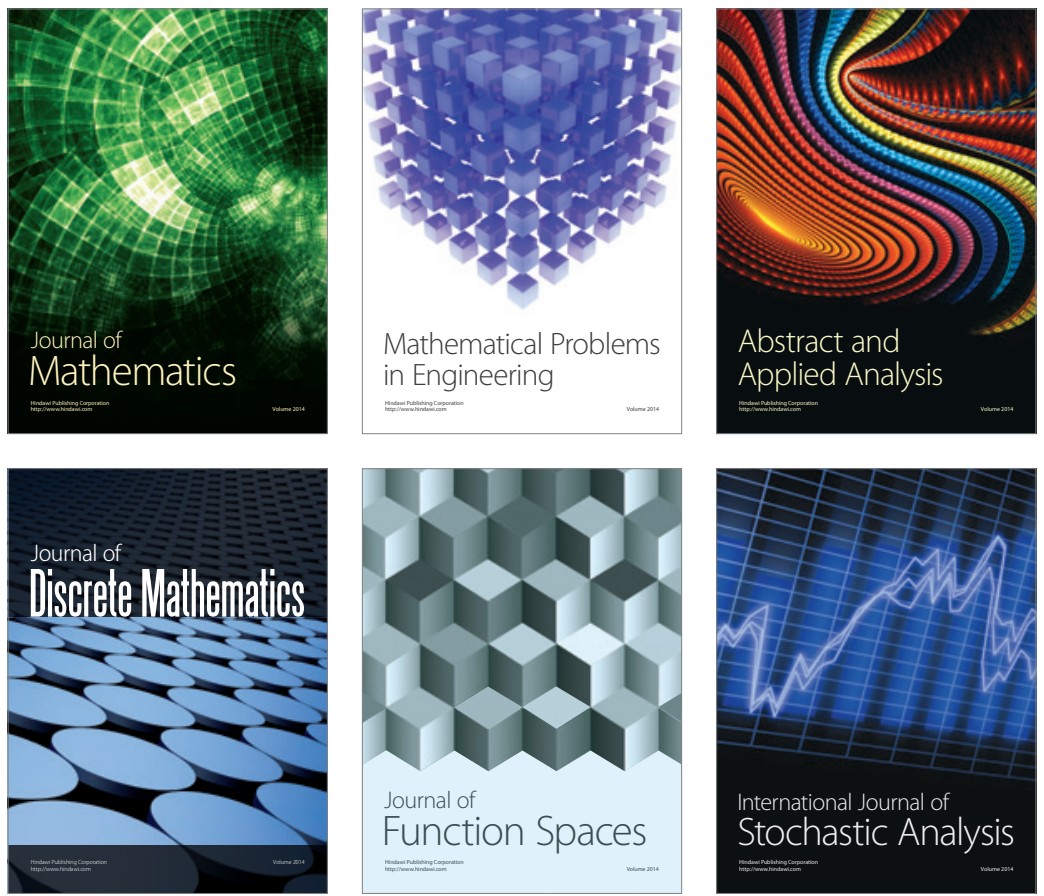

Journal of

Function Spaces

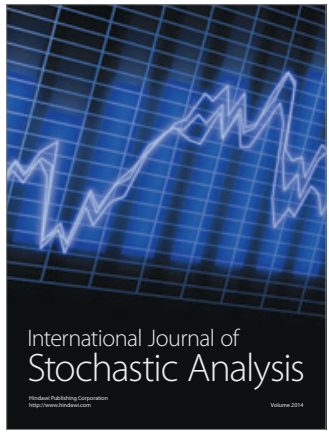

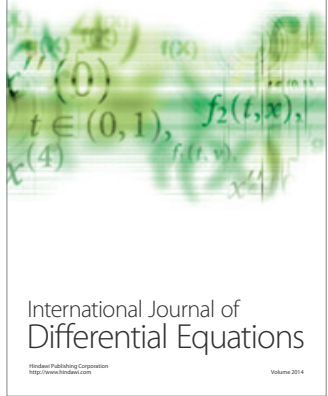
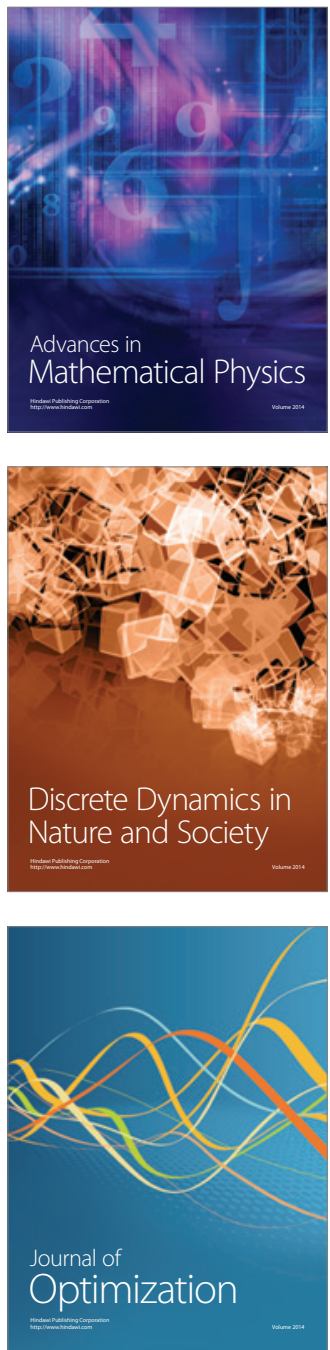